\title{
Right ventricular systolic dysfunction and remodelling in Nigerians with peripartum cardiomyopathy: a longitudinal study
}

\author{
Kamilu Musa Karaye ${ }^{1,2^{*}}$, Krister Lindmark ${ }^{2,3}$ and Michael Henein ${ }^{2,3}$
}

\begin{abstract}
Background: The literature on right ventricular systolic dysfunction (RVSD) in peripartum cardiomyopathy (PPCM) patients is scanty, and it appears that RV reverse remodelling in PPCM has not been previously described. This study thus aimed to assess RVSD and remodelling in a cohort of PPCM patients in Kano, Nigeria.

Methods: A longitudinal study carried out in 3 referral hospitals in Kano, Nigeria. Consecutive PPCM patients who had satisfied the inclusion criteria were recruited and followed up for 12 months. RVSD was defined as the presence of either tricuspid annular plane systolic excursion (TAPSE) $<16 \mathrm{~mm}$ or peak systolic wave $\left(\mathrm{S}^{\prime}\right)$ tissue Doppler velocity of RV free wall $<10 \mathrm{~cm} / \mathrm{s}$. For the purpose of this study, recovery of RV systolic function was defined as an improvement of reduced TAPSE to $\geq 16 \mathrm{~mm}$ or $S^{\prime}$ to $\geq 10 \mathrm{~cm} / \mathrm{s}$, without falling to reduced levels again, during follow-up.

Results: A total of 45 patients were recruited over 6 months with a mean age of $26.6 \pm 7.0$ years. RV systolic function recovery occurred in a total of 8 patients $(8 / 45 ; 17.8 \%)$, of whom $6(75.0 \%)$ recovered in 6 months after diagnosis. The prevalence of RVSD fell from $71.1 \%$ at baseline to $36.4 \%$ at 6 months ( $p=0.007)$ and $18.8 \%$ at 1 year ( $p=0.0008$ vs baseline; $p=0.41$ vs 6 month). Patients with RVSD had higher serum creatinine, and TAPSE accounted for $19.2 \%(p=0.008)$ of the variability of serum creatinine at 6 months. Although $83.3 \%$ of the deceased had RVSD, it didn't predict mortality in the regression models ( $p>0.05$ ).

Conclusion: RVSD and reverse remodelling were common in Nigerians with PPCM, in whom the first 6 months after diagnosis seem to be critical for RV recovery and survival.
\end{abstract}

Keywords: Peripartum cardiomyopathy, Right ventricular dysfunction, RV remodelling

\section{Background}

Peripartum cardiomyopathy (PPCM) is an important cause of heart failure (HF) in many parts of the world including Northern Nigeria, and is associated with significant morbidity and mortality $[1,2]$. We previously described right ventricular (RV) systolic dysfunction in PPCM patients using tricuspid annular plane systolic excursion (TAPSE), and reported RV systolic dysfunction (RVSD) in $54.6 \%$ of the patients [3]. It is believed that left ventricular (LV) function recovers in $23-41 \%$ of

\footnotetext{
* Correspondence: kkaraye@yahoo.co.uk

'Department of Medicine, Bayero University and Aminu Kano Teaching Hospital, 3 New Hospital Road, Kano, Nigeria

${ }^{2}$ Department of Public Health and Clinical Medicine, Umea University, SE-901

87 Umea, Sweden

Full list of author information is available at the end of the article
}

PPCM patients over time, but the literature on RVSD in PPCM is still scanty and to the best of our knowledge, RV reverse remodelling in PPCM has not been previously described [2]. We hypothesised that many PPCM patients would also experience RV reverse remodelling over time. The present study thus aimed to assess RVSD and reverse remodelling over 1 year in a cohort of PPCM patients in Kano, Nigeria.

\section{Methods}

This is a longitudinal study carried out in Murtala Mohammed Specialist Hospital (MMSH), Aminu Kano Teaching Hospital (AKTH) and a private cardiology clinic in Kano, Nigeria. 


\section{Clinical evaluation}

The study conformed to the ethical guidelines of the Declaration of Helsinki, on the principles for medical research involving human subjects [4]. The research protocol was approved by the Research Ethics Committees of AKTH and Kano State Hospitals Management Board before the study started. Inclusion criteria were: (i) new diagnosis of PPCM before commencement of medical treatment; (ii) onset of HF symptoms between last few months of pregnancy and first 5 months postpartum, (iii) at least 18 years of age; (iv) contact telephone number, except patients who gave reassurance that they were willing to attend the follow-up, and (v) giving written informed consent. We excluded PPCM patients who were on HF treatment, as well as those who presented more than 5 months since delivery. PPCM was defined according to the recommendations of the HF Association of the European Society of Cardiology Working Group on PPCM, and LV systolic dysfunction was defined as LV ejection fraction (LVEF) $<45 \%$ [2].

At the study sites, physicians and obstetricians were invited to refer all patients with suspected PPCM to the principal investigator (PI) for further evaluation. Patients were then interviewed, clinically evaluated and recruited consecutively. For each subject, a 12-lead electrocardiogram (ECG) at rest and trans-thoracic echocardiogram were carried out by the PI at the study centres according to standard recommendations [5]. The echocardiographic examination was carried out using Sonoscape S8 Doppler Ultrasound System (Shenzhen, China, 2010). Plasma hemoglobin and serum urea, electrolytes and creatinine were measured at the laboratories of AKTH according to standard protocols.

The PI re-evaluated the patients at 6 and 12 months follow-up, using the same protocol as at recruitment including ECG and echocardiographic examinations, but blood tests were not repeated.

\section{Cardiac function assessment}

Echocardiography was performed according to standard recommendations $[5,6]$. RV basal diameter (RVb), Right atrial longitudinal dimension (RAL) and RA end-systolic area (RAA) were measured in each patient. Tricuspid annular plane systolic excursion (TAPSE) was recorded from the apical four-chamber view with the M-mode cursor positioned at the free wall angle of the tricuspid valve (TV) annulus [7]. RV long axis amplitude of motion (i.e. TAPSE) was measured from end-systolic to end-diastolic points [7], and its peak systolic velocity (S') was measured from myocardial tissue Doppler imaging (TDI). All recordings of TAPSE and S' were obtained during held end-expiration. Care was taken to align M-mode or TDI beam along the direction of tricuspid annulus motion, with the minimum angle in between. TDI sample volume was positioned at $10 \mathrm{~mm}$ from the insertion site of the tricuspid leaflets or $10 \mathrm{~mm}$ away within RV lateral wall and adjusted to cover the longitudinal excursion of the tricuspid annulus both in systole and diastole [5]. RVSD was defined as the presence of either TAPSE $<16 \mathrm{~mm}$ or S' of RV lateral tricuspid annulus $<10 \mathrm{~cm} / \mathrm{s}$ [6]. For the purpose of this study, recovery of RV systolic function was defined as an improvement of reduced TAPSE to $\geq 16 \mathrm{~mm}$ or $S^{\prime}$ to $\geq 10 \mathrm{~cm} / \mathrm{s}$, without falling to reduced levels again, during follow-up.

Pulmonary artery systolic pressure (PASP) was estimated using continuous wave Doppler of the maximum velocity of the tricuspid regurgitant jet (v), from which the retrograde pressure drop was calculated using the modified Bernoulli equation $\left(4 \mathrm{~V}^{2}\right)$, and adding to it the estimated right atrial pressure (RAP) [8]. RAP was estimated using the diameter and collapse of the inferior vena cava during spontaneous respiration, as previously described [9]. Pulmonary hypertension (PHT) was defined as mean pulmonary arterial pressure (mPAP) of $\geq 25 \mathrm{mmHg}$ at rest [10]. mPAP was estimated from PASP using the Chemla formula as: $\operatorname{mPAP}=(0.61 \times$ PASP) +2 (mmHg) [11].

\section{Statistical analysis}

Continuous variables were explored for the presence of skewness, which was corrected with logarithmic $\left(\log _{10}\right)$ transformation. Patients' baseline characteristics were described using frequencies and mean, while time period between patients' delivery and recruitment was described using the median and inter-quartile range (IQR). Chi-square, Fisher's exact probability and Student $t$-tests were used to compare categorical and continuous variables as appropriate. Spearman correlation coefficient $\left(\rho_{s}\right)$ was used to assess the association between TAPSE and mPAP and serum creatinine, while logistic regression models were used to assess the associations between RVSD or mortality and variables of interest. Linear regression was also used to assess the relationship between TAPSE and mPAP. Estimates for regression analyses were expressed as Odds Ratios (OR) and $95 \%$ Confidence Intervals (CI). The regression results were tested with Hosmer and Lemeshow's goodness of fit test, and a $p$-value $\geq 0.05$ implied that the model's estimates fit the data at an acceptable level. The statistical analysis was carried out using SPSS version 16.0 software. Twosided $p$-value $<0.05$ was considered as minimum level of statistical significance.

\section{Results}

The study flow is shown in Fig. 1. A total of 71 patients suspected to have PPCM were referred to the PI for possible inclusion, but only 51 (71.8 \%) satisfied the diagnostic criteria, and all of them developed the disease 


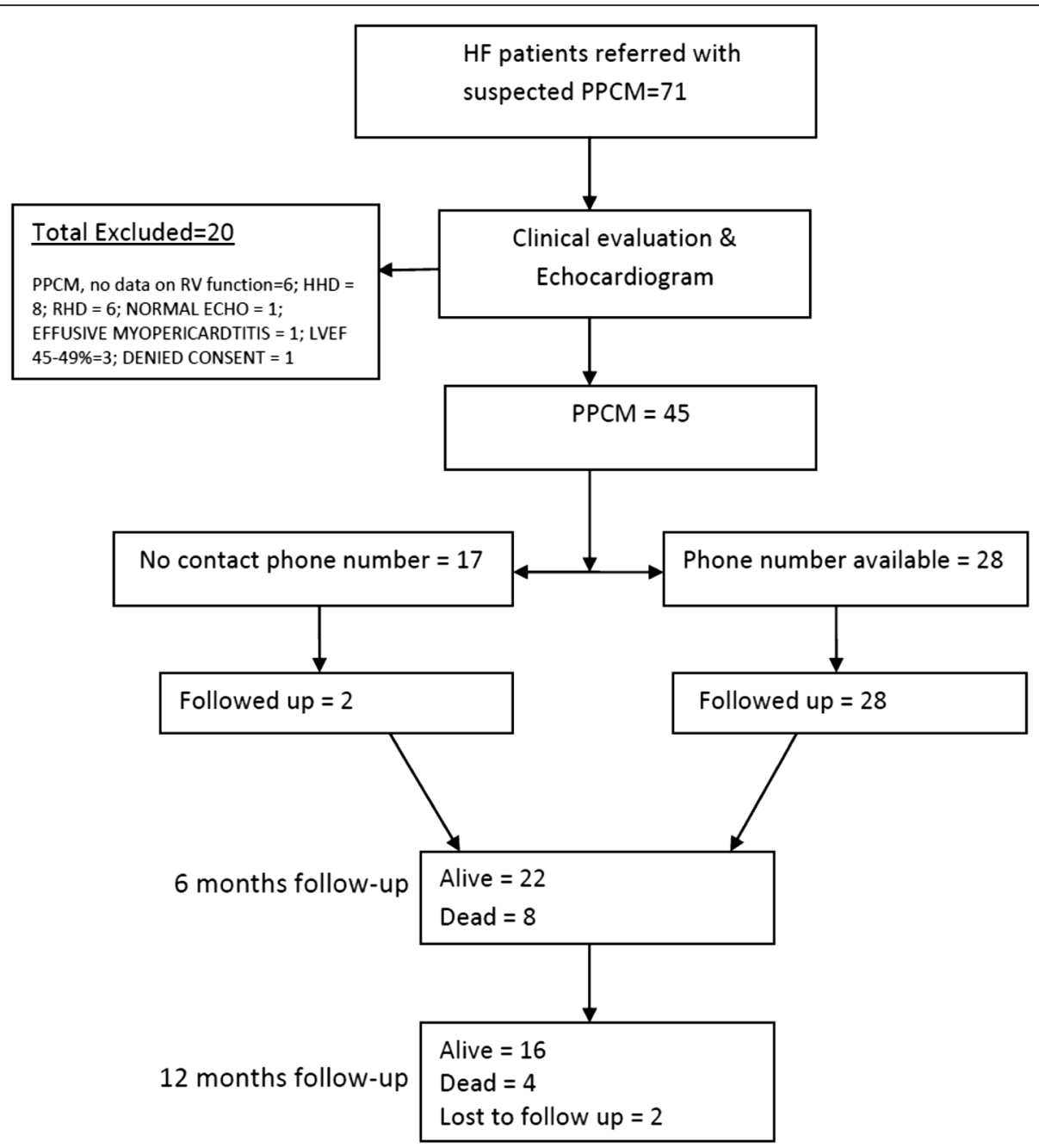

Fig. 1 Flow chart of recruitment and follow-up of patients

postpartum. Of the 51 PPCM patients, 45 (88.2 \%) had complete data for RVSD assessment at baseline, and of these $22(48.9 \%)$ were reviewed at 6 months, 8 (17.8\%) had died, while the remaining 15 (33.3\%) were lost to follow-up. At 1 year, 16/45 (35.6\%) patients were alive, $12(26.7 \%)$ had died and 17 (37.8\%) were lost to followup. The median time from delivery to recruitment for the patients was 6.0 (IQR: 3-15) weeks, and they were recruited in July to December 2013.

\section{Baseline clinical characteristics of patients with and without RVSD (Table 1)}

Of the 45 recruited patients, 32 (71.1\%) had RVSD while the remaining 13 (28.9 \%) had normal RV systolic function. When these 2 groups were compared, the differences were not statistically significant except for higher serum creatinine among those with RVSD $(p=0.02)$, who also tended to have lower diastolic blood pressure (DBP) $(p=0.05)$.

\section{RV remodelling}

Baseline echocardiographic variables were compared between patients with and without RVSD in Table 2. Those with RVSD had significantly lower mean TAPSE and S' $(p<0.001)$, but RV basal diameter, RAA, RAL, mPAP, and prevalence of PHT was not significantly different $(p>0.05)$ between the groups. Mean PAP was $29.3 \pm$ $12.1 \mathrm{mmHg}$ among all subjects respectively, out of whom a total of $30(66.7 \%)$ had PHT.

The pattern of RV reverse remodelling is presented in Fig. 2, which shows increased TAPSE $(p=0.049)$ at 6 months, but RAL $(p=0.04)$ reduced at 12 months and mPAP fell at both $6(p=0.008)$ and 12 months $(p=0.020)$ follow up. The prevalence of RVSD reduced from $71.1 \%$ at baseline to $36.4 \%(8 / 22$ patients $)$ at 6 months $(p=0.007)$ and to $18.8 \%(3 / 16$ patients $)$ at 12 months ( $p=0.001$ vs baseline and $p=0.41$ vs 6 month). In addition, the prevalence of PHT fell to $36.4 \%(p=0.019)$ at 6 months and $31.3 \%(p=0.03)$ 
Table 1 Baseline clinical characteristics of patients with and without RVSD

\begin{tabular}{|c|c|c|c|}
\hline Variables & $\begin{array}{l}\text { Patients with RVSD } \\
N=32(71.1 \%)\end{array}$ & $\begin{array}{l}\text { Patients without RVSD } \\
N=13(28.9 \%)\end{array}$ & $p$-value \\
\hline Age (years) & $26.1 \pm 7.5$ & $27.7 \pm 5.4$ & 0.500 \\
\hline NYHA class: & & & 0.809 \\
\hline$\|$ & $13(40.6 \%)$ & $6(46.2 \%)$ & \\
\hline III & $12(37.5 \%)$ & $5(38.5 \%)$ & \\
\hline IV & $7(21.9 \%)$ & $1(7.7 \%)$ & \\
\hline Parity $\geq 2$ & 25 (78.9\%) & 10 (76.9\%) & 0.758 \\
\hline Breastfeeding & 30 (93.8 \%) & $13(100 \%)$ & - \\
\hline BMI $\left(\mathrm{Kg} / \mathrm{m}^{2}\right)$ & $21.4 \pm 4.6$ & $21.9 \pm 3.9$ & 0.704 \\
\hline Systolic BP (mmHg) & $116 \pm 23$ & $128 \pm 26$ & 0.172 \\
\hline Diastolic BP (mmHg) & $83 \pm 18$ & $94 \pm 16$ & 0.051 \\
\hline Heart rate/min & $111 \pm 16$ & $104 \pm 21$ & 0.302 \\
\hline Pedal oedema & $26(81.3 \%)$ & $8(61.5 \%)$ & 0.312 \\
\hline Hepatomegaly & 19 (59.4 \%) & $6(46.2 \%)$ & 0.515 \\
\hline Pregnancy associated hypertension & $13(40.6 \%)$ & $9(69.2 \%)$ & 0.158 \\
\hline Haemoglobin (g/dL) & $12.2 \pm 1.8$ & $12.9 \pm 1.3$ & 0.202 \\
\hline $\log _{10}$ Creatinine & $2.0 \pm 0.2$ & $1.9 \pm 0.1$ & $0.020^{*}$ \\
\hline Sodium (mmol/L) & $136.3 \pm 6.3$ & $135.5 \pm 5.2$ & 0.655 \\
\hline \multicolumn{4}{|l|}{ Treatment: } \\
\hline ACEI/ARB & 15 (46.9 \%) & 7 (53.9 \%) & 0.749 \\
\hline Frusemide & 32 (100\%) & 13 (100\%) & - \\
\hline Spironolactone & 31 (96.9 \%) & 11 (84.6 \%) & 0.196 \\
\hline Digoxin & 28 (87.57 \%) & 12 (92.3\%) & $>0.999$ \\
\hline Beta blockers & 2 (6.3\%) & 1 (7.7 \%) & $>0.999$ \\
\hline Warfarin & 1 (3.1\%) & 1 (7.7 \%) & 0.499 \\
\hline a-Methyl Dopa & $4(12.5 \%)$ & 2 (15.4 \%) & $>0.999$ \\
\hline
\end{tabular}

Key: NYHA New York Heart Association functional classification, BMI body mass index, $A C E I$ angiotensin concerting enzyme inhibitors, $A R B$ angiotensin receptor blockers. Results are presented as means \pm standard deviations, or as numbers with percentages in parentheses. ${ }^{*} p$-vlaue is statistically significant

at 12 months. RV systolic function recovery occurred in a total of 8 patients $(8 / 45 ; 17.8 \%)$, of whom 6 $(75.0 \%)$ recovered in 6 months. Improvement in TAPSE alone was observed in 2 patients; in $S^{\prime}$ alone in another 2 patients, while both TAPSE and $S^{\prime}$ improved in 4 other patients.

Further analysis showed that although baseline TAPSE was significantly associated with mPAP at 6 months follow-up $\left(\rho_{\mathrm{s}}=-0.531 ; p=0.023\right)$, it did not predict its variability $\left(\mathrm{R}^{2}=0.217 ; p=0.051\right.$. Baseline TAPSE correlated with $\log _{10}$ creatinine $\left(\rho_{\mathrm{s}}=+0.332 ; p=0.048\right)$, and accounted for $19.2 \%(p=0.008)$ of the variability of serum creatinine (Fig. 3). In addition, RVSD significantly increased the odds for $\log _{10}$ creatinine $>1.95$ (equivalent to serum creatinine $89.1 \mu \mathrm{mol} / \mathrm{l})$ by 5.8 fold $(\mathrm{OR}=5.83$; $\mathrm{CI}=1.263-26.944 ; p=0.024)$.

When the baseline characteristics of subjects followed up were compared with those who were lost, differences between the groups were not statistically significant.

\section{RVSD and mortality}

Of the 30 patients followed-up, 2 (6.7\%) were lost to follow-up and 12 died (40.0 \%), of whom 8 (66.7\%) did so within the first 6 months. The deceased had a median survival time of 19.5 weeks. Of the 12 deceased patients, 10 (83.3 \%) had RVSD while the remaining 2 (16.7\%) had normal RV systolic function $(p=0.47)$. Variables assessed in Tables 1 and 2 were compared between the deceased (12 subjects) and the survivors (16 subjects) at 1 year follow up, and the only significant difference between the groups was a lower serum haemoglobin level in the former $(12.1 \pm 1.3 \mathrm{~g} / \mathrm{dl})$ as compared to the latter $(13.5 \pm 1.4 \mathrm{~g} / \mathrm{dl})(p=0.012)$. Step wise univariate regression analyses were then carried out in which the serum haemoglobin and the other variables in the Tables were assessed for possible association with 1 year mortality. However, the one year mortality wasn't predicted by any variable in the univariate regression models, including RVSD $(p=0.284)$, serum creatinine $(p=0.441)$ 
Table 2 Baseline echocardiographic characteristics of patients with and without RVSD

\begin{tabular}{|c|c|c|c|}
\hline Variables & Patients with RVSD $(N=32)$ & Patients without RVSD $(N=13)$ & $p$-value \\
\hline Right atrial end-systolic area, $\mathrm{cm}^{2}$ & $19.7 \pm 7.6$ & $15.8 \pm 8.1$ & 0.149 \\
\hline Right atrial length, mm & $42.9 \pm 10.2$ & $42.5 \pm 11.1$ & 0.916 \\
\hline Right ventricular basal diameter, $\mathrm{mm}$ & $49.6 \pm 9.3$ & $47.7 \pm 10.7$ & 0.583 \\
\hline TAPSE, mm & $13.1 \pm 2.6$ & $20.3 \pm 2.2$ & $<0.001^{*}$ \\
\hline Right ventricular $S^{\prime}, \mathrm{cm} / \mathrm{s}$ & $10.6 \pm 3.2$ & $15.3 \pm 3.9$ & $<0.001^{*}$ \\
\hline RIMP & $1.09 \pm 0.60$ & $1.76 \pm 1.30$ & $0.032^{*}$ \\
\hline RV wall thickness, mm & $3.6 \pm 1.2$ & $3.5 \pm 0.8$ & 0.952 \\
\hline Tricuspid Valve E:A ratio & $1.21 \pm 0.44$ & $0.90 \pm 0.35$ & $0.019^{*}$ \\
\hline Tricuspid Valve E/e' & $5.08 \pm 2.73$ & $3.46 \pm 1.45$ & $0.023^{*}$ \\
\hline Left atrial diameter, $\mathrm{mm}$ & $43.2 \pm 6.4$ & $41.9 \pm 6.2$ & 0.548 \\
\hline LV end-diastolic diameter, mm & $62.0 \pm 7.5$ & $62.4 \pm 11.7$ & 0.910 \\
\hline LV ejection fraction, $\%$ & $32.4 \pm 9.3$ & $36.6 \pm 7.7$ & 0.127 \\
\hline LV stroke volume index, $\mathrm{L} / \mathrm{m}^{2}$ & $39.9 \pm 12.3$ & $51.4 \pm 17.9$ & 0.017 \\
\hline Anti- $\log _{10}$ mitral valve $\mathrm{E}: \mathrm{A}$ & $1.50 \pm 0.62$ & $1.31 \pm 0.25$ & 0.154 \\
\hline Mitral valve E/e' & $15.96 \pm 7.13$ & $15.61 \pm 7.06$ & 0.882 \\
\hline PA acceleration time, ms & $61.8 \pm 22.4$ & $78.9 \pm 28.0$ & $0.037^{*}$ \\
\hline PA systolic pressure, $\mathrm{mmHg}$ & $47.4 \pm 19.4$ & $39.2 \pm 21.5$ & 0.246 \\
\hline Mean PAP, mmHg & $30.6 \pm 11.6$ & $25.9 \pm 13.1$ & 0.273 \\
\hline Pulmonary hypertension & $23(71.9 \%)$ & $7(53.9 \%)$ & 0.304 \\
\hline
\end{tabular}

Key: TAPSE Tricuspid annular plane systolic excursion, $L V$ left ventricle, $E: A$ transvalvular filling velocities ratio, $E / e^{\prime}$ ratio of early filling to averaged early diastolic tissue Doppler velocities of mitral or tricuspid valves, PAP pulmonary artery pressure, RIMP right ventricular (RV) index of myocardial performance. Results are presented as means \pm standard deviations, or as numbers with percentages in parentheses. ${ }^{*} p$-vlaue is statistically significant

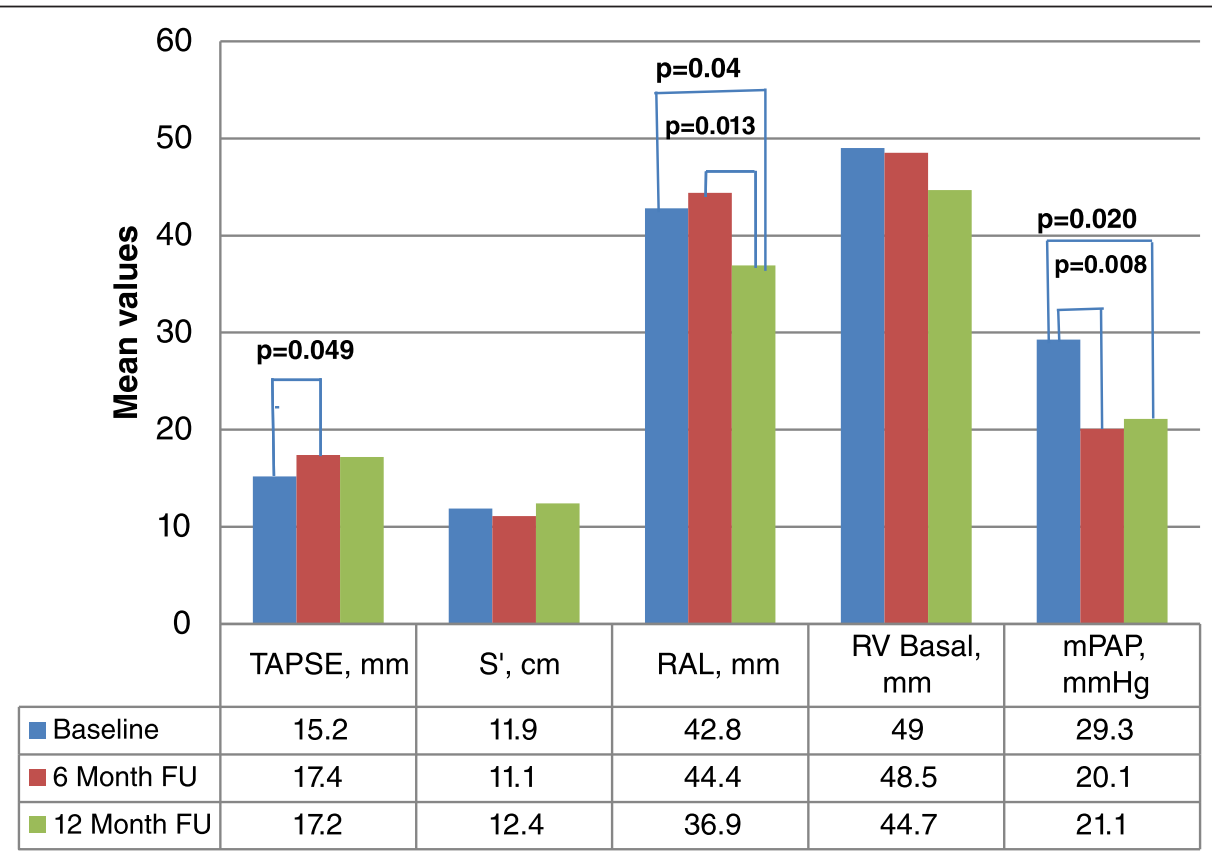

Fig. 2 Pattern of RV remodelling among patients. Legend: TAPSE tricuspid annular plane systolic excursion, RAL right atrial length, RV Basal right ventricular basal diameter, MPAP mean pulmonary artery pressure, FU follow-up. Mean values of variables were computed and compared using Student $t$ - test 


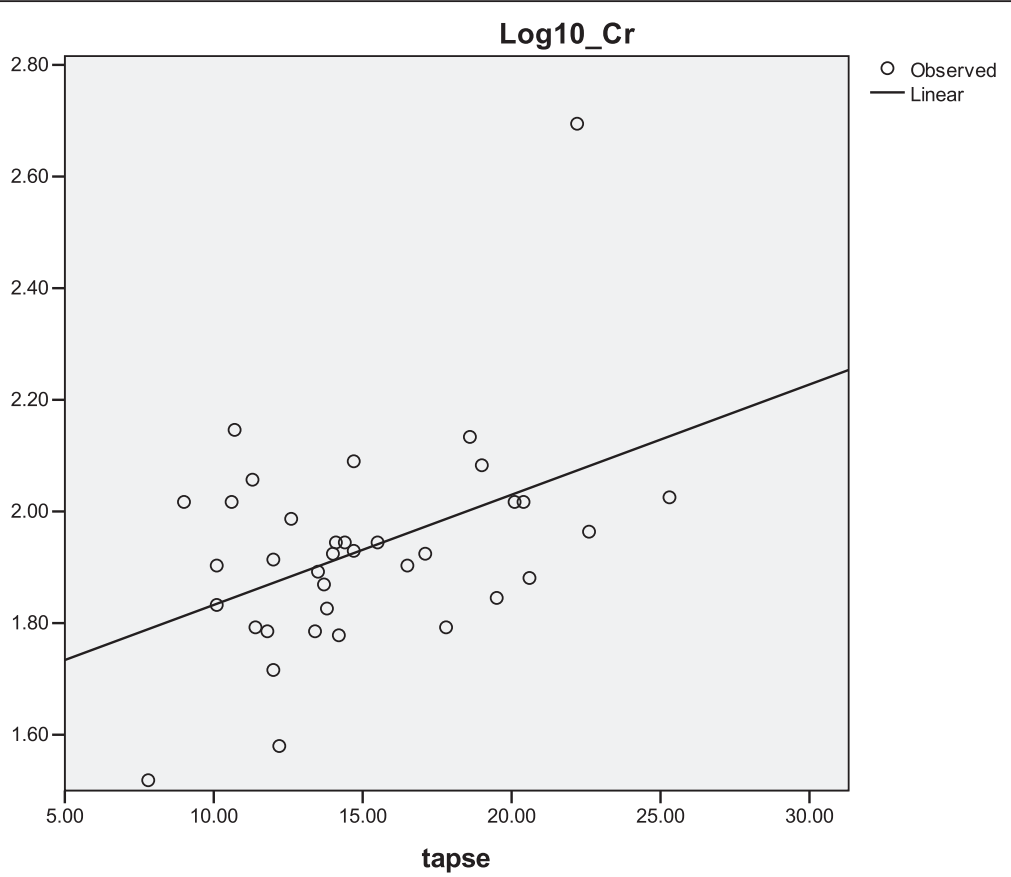

Fig. 3 Relationship between TAPSE and serum creatinine among PPCM patients. Legend: Linear regression model showing that TAPSE accounted for $19.2 \%\left(R^{2}=19.2 \% ; p=0.008\right)$ of the variability of serum creatinine

and haemoglobin $(p=0.053)$ (Hosmer \& Lemeshow test $X^{2}=9.69 ; p=0.288$.

\section{Discussion}

The present longitudinal study assessed RVSD and RV remodelling and its response to treatment and potential recovery in a group of PPCM patients from Kano, Nigeria. The prevalence of RVSD, in the form of reduced TAPSE and RV free wall $S^{\prime}$ velocity was evident in $71.1 \%$ of the patients at baseline, and fell to $36.4 \%$ at 6 months and to $18.8 \%$ at 12 months follow-up. Likewise, PHT was found in $66.7 \%$ of patients at baseline, and persisted in $36.4 \%$ at 6 months, and to $31.3 \%$ at 12 months follow up. RV systolic function recovery occurred in a total of 8 patients $(8 / 45 ; 17.8 \%)$, of whom $6(75.0 \%)$ recovered in 6 months. Forty percent of the followed-up patients died within 1 year; two-thirds of them within the first 6 months after diagnosis.

RVSD, its recovery and potential relationship with mortality are not well studied in PPCM. Based on reduced TAPSE, we have previously reported a prevalence of RVSD of $54.6 \%$ in PPCM patients [3]. Adding RV reduced myocardial velocity $\left(S^{\prime}\right)$ raised the prevalence of patients with RVSD in this study to $71.1 \%$, suggesting a more accurate means for identifying such patients. The second important observation in the current study is the significant recovery of RVSD along with its pressure afterload in the form of PHT. Indeed, 6 months from the time of presentation, the prevalence of RVSD, PHT fell by more than $50 \%$ despite poor adherence to heart failure conventional medications. Thus, the observed RV reverse remodelling seems to be related to the recovery of the pulmonary circulation status rather than to the effect of medications as has been previously observed in the LV [12]. This claim is supported by the modest relationship we found between TAPSE and mPAP at 6 months follow-up. Our finding might be explained by the significant RV function recovery in more than $50 \%$ of patients at 6 months, thus suggesting a different pathophysiologic mechanism to right ventricular - pulmonary circulation coupling in PPCM compared with DCM, which has a more chronic course of disturbances of ventricular function [13]. In addition, TAPSE was linearly related to serum creatinine, confirming the adverse relationship between RVSD and renal impairment $[14,15]$. Whether this reflects the aggressive nature of cardiac decompensation in PPCM, since all the patients developed LV systolic dysfunction with LVEF $<45 \%$ within a few postpartum days, remains to be verified in a larger group of patients.

Thus, our findings show that the first 6 months of PPCM diagnosis seem to be crucial for RV function recovery as well as survival. These findings are supported by Whitehead et al. who reported $87 \%$ of PPCM deaths occurring within the first 6 months of diagnosis, and Elkayam who observed that LV function normalises in 
approximately $50 \%$ of women with PPCM within the same period [12, 16]. These findings urge intensive medical treatment for such young patients within the first few days and weeks of their presentation. Finally, our mortality rate of $40 \%$ in the present study seems high compared to $13 \%$ over 6 months in South Africa [17]. This could be partly explained by the high maternal mortality in Nigeria in general, which was as high as 496.4 per 100,000 live births in 2013 [18].

\section{Clinical implications}

In the absence of magnetic resonance imaging, which is known for its superior accuracy in assessing RV structure and function, bedside echocardiography has an acceptable accuracy for diagnosing and monitoring RV function in PPCM patients, and it is widely available and affordable, particularly in countries where PPCM is relatively more prevalent [19]. With the high mortality recorded in the present study, PPCM patients should receive intensive care in the acute phase, with thorough assessment and follow up of $\mathrm{LV}$ as well as RV function in an attempt to identify the vulnerable ones who need closer follow up.

\section{Study limitations}

Although important, B-type natriuretic peptide (BNP) or $\mathrm{N}$-terminal pro-BNP and other biomarkers could not be assessed because the tests were not readily available at the study centres. Secondly, it is important to point out that 15 of the 45 patients could not be followed-up because they were not contactable in spite of adequate counselling. Their inability to come for follow-up isn't unrelated to the significant security challenges being faced by northern Nigeria including Kano, making movements and keeping to appointments extremely difficult. Thirdly, cardiac magnetic resonance imaging could have shed more light on the nature of the myocardial pathology in PPCM, but this facility was not available at the study centres [19].

\section{Conclusion}

The present study assessed RVSD and pulmonary circulation disturbances in a group of PPCM patients in Kano, Nigeria. These were prevalent in over two-thirds of the patients at presentation and improved by more than $50 \%$ at 6 months despite the lack of adherence to medical therapy. However, the studied cohort suffered a relatively high mortality which was not predicted by RVSD or other variables, in keeping with the overall high maternal mortality in Nigeria. These findings urge intensive care for such young patients during the acute phase of the disease to support cardiac function recovery and reduce mortality.

\section{Abbreviations}

PPCM: peripartum cardiomyopathy; HF: heart failure; RV: right ventricle; TAPSE: tricuspid annular plane systolic excursion; RVSD: right ventricular systolic dysfunction; LV: left ventricle; MMSH: Murtala Mohammed specialist hospital; AKTH: Aminu Kano teaching hospital; LVEF: LV ejection fraction; PI: principal investigator; ECG: electrocardiogram; RAA: right atrial area; RVb: RV basal dimension; RAL: RA length; TV: tricuspid valve; TDI: tissue Doppler imaging; PASP: pulmonary artery systolic pressure; RAP: RA pressure; PHT: pulmonary hypertension; mPAP: mean pulmonary artery pressure; RIMP: right ventricular index of myocardial performance; $\rho_{s}$ : Spearman correlation coefficient; OR: odds ratio; Cl: confidence interval; DBP: diastolic blood pressure; BNP: B-type natriuretic peptide.

\section{Competing interest}

The authors hereby declare that we have no competing interests regarding this manuscript.

\section{Authors' contributions}

KMK conceptualised and designed the study, and acquired, analysed and interpreted data, drafted the article and revised it critically for important intellectual content, and approved the final submitted version. $\mathrm{KL}$ analysed and interpreted data, revised the article critically for important intellectual content, and approved the final submitted version. MYH designed the study, analysed and interpreted data, revised the article critically for important intellectual content, and approved the final submitted version.

\section{Author details}

${ }^{1}$ Department of Medicine, Bayero University and Aminu Kano Teaching Hospital, 3 New Hospital Road, Kano, Nigeria. ${ }^{2}$ Department of Public Health and Clinical Medicine, Umea University, SE-901 87 Umea, Sweden.

${ }^{3}$ Department of Cardiology, Umea Heart Centre, SE-901 87 Umea, Sweden.

Received: 15 October 2015 Accepted: 22 January 2016

Published online: 29 January 2016

\section{References}

1. Karaye KM, Sani MU. Factors associated with poor prognosis among patients admitted with heart failure in a Nigerian tertiary medical centre: a crosssectional study. BMC Cardiovasc Disord. 2008;8(1):16.

2. Sliwa K, Hilfiker-Kleiner D, Petrie MC, Mebazaa A, Pieske B, Buchmann E, et al. Heart Failure Association of the European Society of Cardiology Working Group on Peripartum Cardiomyopathy. Current state of knowledge on aetiology, diagnosis, management and therapy of peripartum cardiomyopathy: a position statement from the Heart Failure Association of the European Society of Cardiology Working Group on peripartum cardiomyopathy. Eur J Heart Fail. 2010;12(8):767-78.

3. Karaye KM. Right ventricular systolic function in peripartum and dilated cardiomyopathies. Eur J Echocardiogr. 2011;12(5):372-4.

4. World Medical Association Declaration of Helsinki. Ethical Principles for Medical Research Involving Human Subjects. J Postgrad Med. 2002;48:206-8.

5. Lang RM, Badano LP, Mor-Avi V, Afilalo J, Armstrong A, Ernande L, et al. Recommendations for cardiac chamber quantification by echocardiography in adults: an update from the American Society of Echocardiography and the European Association of Cardiovascular Imaging. J Am Soc Echocardiogr. 2015;28(1):1-39.

6. Rudski LG, Lai WW, Afilalo J, Hua L, Handschumacher MD, Chandrasckaran K, et al. Guidelines for the echocardiographic assessment of the right heart in adults: a report from the American Society of echocardiography. Endorsed by the European Society of Cardiology, and the Canadian Society of echocardiography. J Am Soc Echocardiogr. 2010;23:685-713.

7. Lindqvist $P$, Henein $M$, Kazzam $E$. Right ventricular outflow tract fractional shortening: an applicable measure of right ventricular systolic function. Eur J Echocardiogr. 2003;4:29-35.

8. Sciomer S, Magri D, Badagliacca R. Non-invasive assessment of pulmonary hypertension: Doppler echocardiography. Pulm Pharmacol Ther. 2007;20:135-40.

9. Posteraro A, Salustri A, Trambaiolo P, Amici E, Gambelli G. Echocardiographic estimation of pulmonary pressures. J Cardiovasc Med. 2006;7:545-54.

10. Galie' N, Hoeper MM, Humbert M, Torbicki A, Vachiery JL, Barbera JA, et al. Guidelines for the diagnosis and treatment of pulmonary hypertension. The 
Task Force for the Diagnosis and Treatment of Pulmonary Hypertension of the European Society of Cardiology (ESC) and the European Respiratory Society (ERS), endorsed by the International Society of Heart and Lung Transplantation (ISHLT). Eur Heart J. 2009:30:2493-537.

11. Chemla D, Castelain V, Humbert M, Hébert JL, Simonneau G, Lecarpentier Y, et al. New Formula for Predicting Mean Pulmonary Artery Pressure Using Systolic Pulmonary Artery Pressure. Chest. 2004;126:1313-7.

12. Elkayam U. Clinical characteristics of peripartum cardiomyopathy in the United States: diagnosis, prognosis, and management. J Am Coll Cardiol. 2011;58(7):659-70.

13. Di Mauro M, Calafiore AM, Penco M, Romano S, Di Giammarco G, Gallina S. Mitral valve repair for dilated cardiomyopathy: predictive role of right ventricular dysfunction. Eur Heart J. 2007;28(20):2510-6.

14. Mullens W, Abrahams Z, Francis GS, Sokos G, Taylor DO, Starling RC, et al. Importance of venous congestion for worsening of renal function in advanced decompensated heart failure. J Am Coll Cardiol. 2009;53(7):589-96.

15. Guinot $\mathrm{PG}$, Arab OA, Longrois $\mathrm{D}$, Dupont $\mathrm{H}$. Right ventricular systolic dysfunction and vena cava dilatation precede alteration of renal function in adult patients undergoing cardiac surgery: an observational study. Eur J Anaesthesiol. 2014;31:1-8.

16. Whitehead SJ, Berg CJ, Chang J. Pregnancy-related mortality due to cardiomyopathy: United States, 1991-1997. Obstet Gynecol. 2003;102:1326-31.

17. Blauwet LA, Libhaber E, Forster O, Tibazarwa K, Mebazaa A, Hilfiker-Kleiner D, et al. Predictors of outcome in 176 South African patients with peripartum cardiomyopathy. Heart. 2013;99(5):308-13.

18. Kassebaum NJ, Bertozzi-Villa A, Coggeshall MS, Shackelford KA, Steiner C, Heuton KR, et al. Global, regional and national levels and causes of maternal mortality during 1990-2013: a systematic analysis for the Global Burden of Disease Study 2013. Lancet. 2014;384(9947):980-1004.

19. Blecker GB, Steendijk P, Holman ER, Yu C-M, Breithardt OA, Kaandorp TAM, et al. Assessing right ventricular function: the role of echocardiography and complementary technologies. Heart. 2006;2(Suppl I):i19-26.

\section{Submit your next manuscript to BioMed Central and we will help you at every step:}

- We accept pre-submission inquiries

- Our selector tool helps you to find the most relevant journal

- We provide round the clock customer support

- Convenient online submission

- Thorough peer review

- Inclusion in PubMed and all major indexing services

- Maximum visibility for your research

Submit your manuscript at wuw biomedcentral.com/submit

) Biomed Central 